\title{
Complex Number and its Conjugate in Continued Fraction
}

\section{A. Gnanam, S. Krithika}

ABSTRACT: For any equation complex roots occur in pairs. For finding a root of the equation in continued fractions algorithms are available. But to get complex roots in continued fraction no such procedure so far we have. Here, in this paper if $\alpha+i \beta$ has a representation in continued fractions we try to find its conjugate $\alpha-i \beta$ in terms of continued fractions. This will be useful in finding complex roots of a quadratic equations in continued fraction.

KEYWORDS: Continued Fraction, Complex Continued Fraction, Complex numbers.

\section{INTRODUCTION}

An expression of the form

$$
a_{0}+\frac{e_{0}}{a_{1}+\frac{e_{1}}{a_{2}+\frac{e_{2}}{a_{3}+\frac{e_{3}}{\ddots+\frac{e_{n}}{a_{n}+\cdot} .}}}}
$$

Where $a_{0}, a_{1}, a_{2}, a_{3}, \cdots$ are in $Z(i)$ and $e_{i}{ }^{\prime} s$ are units of complex numbers. Therefore

$e_{k} \in\{1,-1, i,-i\}, k=1,2,3, \ldots$ is known as a complex

continued fraction.[1,2,3,4]

Therefore $a_{0}+\frac{e_{1}}{a_{1}+a_{2}+e_{3}+} \frac{e_{3}}{a_{n}} \ldots \frac{e_{n}}{a_{n}}$ is known as finite complex continued fraction and an expression

$$
a_{0}+\frac{e_{1}}{a_{1}+a_{2}+e_{3}+} \frac{e_{3}}{a_{n}+a_{n+1}+} \cdots \frac{e_{n}}{a_{n+1}} \cdot \cdots \text { is known }
$$

as an infinite complex continued fraction. In a finite or infinite complex continued fraction $a_{0}, a_{1}, a_{2}, a_{3}, \cdots$ are called the partial quotients where as $e_{1}, e_{2}, e_{3}, \cdots$ are known as the partial numerators.

1. Algorithm of complex continued fraction: $[3,4]$

Revised Manuscript Received on November 08, 2019.

A.Gnanam, Assistant professor ,Department of Mathematics, Government Arts College, Trichy-22. gnaanam@yahoo.com

S.Krithika, Assistant professor ,Department of Mathematics, Seethalakshmi Ramaswami College, Trichy-2 Email:krithikasrc@gmail.com
Let $x \in C$.Suppose we wish to find continued fraction expansion of $X$, take $x_{0}=\operatorname{Re}(x)+i \operatorname{Im}(x)$.

Let $a=\operatorname{Re}(x)-[\operatorname{Re}(x)]$ and $b=\operatorname{Im}(x)-[\operatorname{Im}(x)]$ so that $a<0$ and $b<0$.

Now $\left[x_{0}\right]=[\operatorname{Re}(x)]+i[\operatorname{Im}(x)]+\alpha$, a floor function.

Where $\alpha=\left\{\begin{array}{cc}0 & \text { if } a+b<0 \\ 1 & \text { if } a+b \geq 1 \text { with } a \geq b \\ i \quad & \text { if } a+b \geq 1 \text { with } a<b\end{array}\right.$

Set $\quad a_{0}=\left[x_{0}\right]$. Then $x_{1}=\frac{1}{x_{0}-\left[x_{0}\right]}$ and

$\left[x_{1}\right]=\left[\operatorname{Re}\left(x_{1}\right)\right]+i\left[\operatorname{Im}\left(x_{1}\right)\right]+\alpha$.

Again set $a_{1}=\left[x_{1}\right]$. Then $x_{2}=\frac{1}{x_{1}-\left[x_{1}\right]}$ and

$\left[x_{2}\right]=\left[\operatorname{Re}\left(x_{2}\right)\right]+i\left[\operatorname{Im}\left(x_{2}\right)\right]+\alpha, a_{2}=\left[x_{2}\right]$ Then

$x_{3}=\frac{1}{x_{2}-\left[x_{2}\right]}$ and $\left[x_{3}\right]=\left[\operatorname{Re}\left(x_{3}\right)\right]+i\left[\operatorname{Im}\left(x_{3}\right)\right]+\alpha$,

$a_{3}=\left[x_{3}\right] \ldots a_{k-1}=\left[x_{k-1}\right]$.

Then $x_{k}=\frac{1}{x_{k-1}-\left[x_{k-1}\right]}$ and

$\left\lfloor x_{k}\right\rfloor=\left\lfloor\operatorname{Re}\left(x_{k}\right)\right\rfloor+i\left\lfloor\operatorname{Im}\left(x_{k}\right)\right\rfloor+\alpha$.and $a_{k}=\left\lfloor x_{k}\right\rfloor$.

The algorithm terminates if the complex continued fraction is finite otherwise it is no terminating.

Theorem

If the complex continued fraction of

$\alpha+i \beta=\left\langle\alpha_{0}+i \beta_{0}, \alpha_{1}, \alpha_{2}+i \beta_{2}, \alpha_{3}+i \beta_{3}\right.$,

$$
\left.\alpha_{4}+i \beta_{4}, \ldots\right\rangle
$$

such that $\alpha, \beta \in Q$ the set of all rational numbers then the complex continued fraction of

$$
\begin{gathered}
\alpha-i \beta=\left\langle\alpha_{0}-i \beta_{0}, \alpha_{1}-i, \alpha_{2}+i \beta_{2}, \alpha_{3}+i \beta_{3},\right. \\
\left.\alpha_{4}+i \beta_{4}, \ldots\right\rangle
\end{gathered}
$$

$\alpha_{i^{\prime}}{ }^{\prime} s, \beta_{i}{ }^{\prime} s$ are integers.

Proof: 


\section{Complex Number and its Conjugate in Continued Fraction}

Taking $\alpha_{k}+i \beta_{k}=c_{k}, k=0,1,2,3 \ldots$,

$$
\begin{aligned}
& c_{i} c_{j} c_{l} c_{m} c_{n}=\left(\alpha_{i} \alpha_{j} \alpha_{l} \alpha_{m} \alpha_{n}-\alpha_{i} \alpha_{j} \beta_{l} \beta_{m} \alpha_{n}\right. \\
& -\alpha_{i} \beta_{j} \alpha_{l} \beta_{m} \alpha_{n}-\alpha_{i} \beta_{j} \beta_{l} \alpha_{m} \alpha_{n}-\alpha_{i} \beta_{j} \alpha_{l} \alpha_{m} \beta_{n} \\
& +\alpha_{i} \beta_{j} \beta_{l} \beta_{m} \beta_{n}-\alpha_{i} \alpha_{j} \beta_{l} \alpha_{m} \beta_{n}-\alpha_{i} \alpha_{j} \alpha_{l} \beta_{m} \beta_{n} \\
& -\beta_{i} \beta_{j} \alpha_{l} \alpha_{m} \alpha_{n}+\beta_{i} \beta_{j} \beta_{l} \beta_{m} \alpha_{n}-\beta_{i} \alpha_{j} \beta_{l} \alpha_{m} \alpha_{n}- \\
& \beta_{i} \alpha_{j} \alpha_{l} \beta_{m} \alpha_{n}-\beta_{i} \alpha_{j} \alpha_{l} \alpha_{m} \beta_{n}+\beta_{i} \alpha_{j} \beta_{l} \beta_{m} \beta_{n} \\
& \left.+\beta_{i} \beta_{j} \alpha_{l} \beta_{m} \beta_{n}+\beta_{i} \beta_{j} \beta_{l} \alpha_{m} \beta_{n}\right)+i\left(\alpha_{i} \beta_{j} \alpha_{l} \alpha_{m} \alpha_{n}\right. \\
& -\alpha_{i} \beta_{j} \beta_{l} \beta_{m} \alpha_{n}+\alpha_{i} \alpha_{j} \beta_{l} \alpha_{m} \alpha_{n}+\alpha_{i} \alpha_{j} \alpha_{l} \beta_{m} \alpha_{n} \\
& +\alpha_{i} \alpha_{j} \alpha_{l} \alpha_{m} \beta_{n}-\alpha_{i} \alpha_{j} \beta_{l} \beta_{m} \beta_{n}-\alpha_{i} \beta_{j} \alpha_{l} \beta_{m} \beta_{n} \\
& -\alpha_{i} \beta_{j} \beta_{l} \alpha_{m} \beta_{n}+\beta_{i} \alpha_{j} \alpha_{l} \alpha_{m} \alpha_{n}-\beta_{i} \alpha_{j} \beta_{l} \beta_{m} \alpha_{n} \\
& -\beta_{i} \beta_{j} \alpha_{l} \beta_{m} \alpha_{n}-\beta_{i} \beta_{j} \beta_{l} \alpha_{m} \alpha_{n}-\beta_{i} \beta_{j} \alpha_{l} \alpha_{m} \beta_{n} \\
& \left.+\beta_{i} \beta_{j} \beta_{l} \beta_{m} \beta_{n}-\beta_{i} \alpha_{j} \beta_{l} \alpha_{m} \beta_{n}-\beta_{i} \alpha_{j} \alpha_{l} \beta_{m} \beta_{n}\right)
\end{aligned}
$$

$$
\frac{p_{3}}{q_{3}}=\left\langle c_{0}, c_{1}, c_{2}, c_{3}\right\rangle=\frac{c_{0} c_{1} c_{2} c_{3}+c_{0} c_{1}+c_{0} c_{3}+c_{2} c_{3}+1}{c_{1} c_{2} c_{3}+c_{1}+c_{3}}
$$

II. RESULT ANALYSIS:

$\frac{p_{4}}{q_{4}}=\left\langle c_{0}, c_{1}, c_{2}, c_{3}, c_{4}\right\rangle$

$c_{0} c_{1} c_{2} c_{3} c_{4}+c_{0} c_{1} c_{2}+c_{0} c_{1} c_{4}+c_{0} c_{3} c_{4}+$$$
=\frac{c_{2} c_{3} c_{4}+c_{0}+c_{2}+c_{4}}{c_{1} c_{2} c_{3} c_{4}+c_{1} c_{2}+c_{1} c_{4}+c_{3} c_{4}+1}
$$

Taking $c_{k}=\alpha_{k}+i \beta_{k}, k=0,1,2,3 \ldots, \quad$ then

For every $\quad i, j=0,1,2,3, \ldots$

$$
c_{i} c_{j}=\left(\alpha_{i} \alpha_{j}-\beta_{i} \beta_{j}\right)+i\left(\alpha_{i} \beta_{j}-\beta_{i} \alpha_{j}\right)
$$

For every $\quad i, j, l=0,1,2,3, \ldots$

$$
\begin{aligned}
& c_{i} c_{j} c_{l}=\left(\alpha_{i} \alpha_{j} \alpha_{l}-\alpha_{i} \beta_{j} \beta_{l}-\beta_{i} \alpha_{j} \beta_{l}-\beta_{i} \beta_{j} \alpha_{l}\right) \\
& +i\left(\beta_{i} \alpha_{j} \alpha_{l}-\beta_{i} \beta_{j} \beta_{l}+\alpha_{i} \beta_{j} \alpha_{l}+\alpha_{i} \alpha_{j} \beta_{l}\right)
\end{aligned}
$$

For every $\quad i, j, l, m=0,1,2,3, \ldots$

$c_{i} c_{j} c_{l} c_{m}=\left(\alpha_{i} \alpha_{j} \alpha_{l} \alpha_{m}-\alpha_{i} \beta_{j} \beta_{l} \alpha_{m}-\beta_{i} \alpha_{j} \beta_{l} \alpha_{m}\right.$

$-\beta_{i} \beta_{j} \alpha_{l} \alpha_{m}-\beta_{i} \alpha_{j} \alpha_{l} \beta_{m}+\beta_{i} \beta_{j} \beta_{l} \beta_{m}-\alpha_{i} \beta_{j} \alpha_{l} \beta_{m}$

$\left.-\alpha_{i} \alpha_{j} \beta_{l} \beta_{m}\right)$

$+i\left(\beta_{i} \alpha_{j} \alpha_{l} \alpha_{m}-\beta_{i} \beta_{j} \beta_{l} \alpha_{m}+\alpha_{i} \beta_{j} \alpha_{l} \alpha_{m}+\alpha_{i} \alpha_{j} \beta_{l} \alpha_{m}+\right.$

$\left.\alpha_{i} \alpha_{j} \alpha_{l} \beta_{m}-\alpha_{i} \beta_{j} \beta_{l} \beta_{m}-\beta_{i} \alpha_{j} \beta_{l} \beta_{m}-\beta_{i} \beta_{j} \alpha_{l} \beta_{m}\right)$

For every $\quad i, j, l, m, n=0,1,2,3, \ldots$
Consider the complex numbers $\alpha \pm i \beta=1.1 \pm 0.9950 i$

\section{Using Continued fraction algorithm: \\ Continued fraction of the complex number $\alpha+i \beta=1.1+0.9950 i$}

Take $x_{0}=1.1+0.9950 i$

Then $a=1.1-(1)=0.1000$ and $b=0.9950-(0)=0.9950$

Therefore $\left[x_{0}\right]=1+0+i=1+i \Rightarrow a_{0}=1+i$.

Now

$$
x_{1}=\frac{1}{[(1.1+0.9950 i)-(1+i)]} \Rightarrow x_{1}=9.9751+0.4988 i
$$

Round off the imaginary part to $0.5, x_{1}=9.9751+0.5 i$

Then $a=9.9751-(9)=0.9751$ and $b=0.5-(0)=0.5$

Therefore $\left[x_{1}\right]=9+0+1=10 \Rightarrow a_{1}=10$.

Again

$$
x_{2}=\frac{1}{[(9.9751+0.5 i)-(10)]} \Rightarrow x_{2}=-0.0993-1.9951 i
$$


Then $a=-0.0993-(-1)=0.0007$

$b=-1.9951-(-2)=0.0049$

Therefore $\left[x_{2}\right]=-1-2 i+0=-1-2 i \Rightarrow a_{2}=-1-2 i$. $x_{3}=\frac{1}{[(-0.0993-1.9951 i)-(-1-2 i)]} \Rightarrow x_{3}=1.1102-0.0060 i$

Then $a=1.1102-(1)=0.1102$

$b=-0.0060-(-1)=0.9940$

Therefore $\left[x_{3}\right]=1-i+i=1 \Rightarrow a_{3}=1$.

$x_{4}=\frac{1}{[(1.1102-0.0060 i)-(1)]} \Rightarrow x_{4}=9.0476+0.4926 i$

Then $a=9.0476-(9)=0.0476$

$b=0.4926-(0)=0.4926$

Therefore $\left[x_{4}\right]=9+0+0=9 \Rightarrow a_{4}=9$.

$x_{5}=\frac{1}{[(9.0476+0.4926 i)-(9)]} \Rightarrow x_{5}=0.1943-2.0113 i$

Then $a=0.1943-(0)=0.1943$

and

$b=-2.0113-(-3)=0.9887$

Therefore $\left[x_{5}\right]=0-3 i+i=-2 i \Rightarrow a_{5}=-2 i$

$$
\begin{aligned}
x_{6} & =\frac{1}{[(0.1943-2.0113 i)-(-2 i)]} \\
& \Rightarrow x_{6}=5.1293+0.2983 i
\end{aligned}
$$

Then $a=5.1293-(5)=0.1293$

$b=0.2983-(0)=0.2983$

Therefore $\left[x_{6}\right]=5+0+0=5 \Rightarrow a_{6}=5$.

$$
x_{7}=\frac{1}{[(5.1293+0.2983)-(5)]} \Rightarrow x_{7}=1.2233-2.8221 i
$$

Then $a=1.2233-(1)=0.2233$

and $b=-2.8221-(-3)=0.4012$

Therefore $\left[x_{7}\right]=1-3 i+0=1-3 i \Rightarrow a_{7}=1-3 i$.

and

and

and

$$
\begin{aligned}
x_{8}= & \frac{1}{[(1.2233-2.8221 i)-(1-3 i)]} \\
& \Rightarrow x_{8}=2.7395-2.1825 i
\end{aligned}
$$

Then $a=2.7395-(2)=0.7395$

and

$b=-2.1825-(-3)=0.8125$

Therefore $\left[x_{8}\right]=2-3 i+i=2-2 i \Rightarrow a_{8}=2-2 i$.

\section{Continued fraction of the complex number $\alpha-i \beta=1.1-0.9950 i$}

Take $x_{0}=1.1-0.9950 i$

Then $a=1.1-(1)=0.1000$

and $b=-0.9950-(-1)=0.0050$

Therefore $\left[x_{0}\right]=1-i+0=1-i \Rightarrow a_{0}=1-i$.

Now

$x_{1}=\frac{1}{[(1.1-0.9950 i)-(1-i)]} \Rightarrow x_{1}=9.9751-0.4988 i$

Round off the imaginary part to $0.5, x_{1}=9.9751-0.5 i$

Then $a=9.9751-(9)=0.9751$

and

$b=-0.5-(-1)=0.5$

Therefore $\left[x_{1}\right]=9-i+1=10-i \Rightarrow a_{1}=10-i$.

Again

$x_{2}=\frac{1}{[(9.9751-0.5 i)-(10-i)]} \Rightarrow x_{2}=-0.0993-1.9951 i$

Then $a=-0.0993-(-1)=0.0007$

and

$b=-1.9951-(-2)=0.0049$

Therefore $\left[x_{2}\right]=-1-2 i+0=-1-2 i \Rightarrow a_{2}=-1-2 i$.

$x_{3}=\frac{1}{[(-0.0993-1.9951 i)-(-1-2 i)]} \Rightarrow x_{3}=1.1102-0.0060 i$

Then $a=1.1102-(1)=0.1102$

and

$b=-0.0060-(-1)=0.9940$ 


\section{Complex Number and its Conjugate in Continued Fraction}

Therefore $\left[x_{3}\right]=1-i+i=1 \Rightarrow a_{3}=1$.

$x_{4}=\frac{1}{[(1.1102-0.0060 i)-(1)]} \Rightarrow x_{4}=9.0476+0.4926 i$

Then $a=9.0476-(9)=0.0476$

$b=0.4926-(0)=0.4926$

Therefore $\left[x_{4}\right]=9+0+0=7 \Rightarrow a_{4}=9$.

$$
\begin{gathered}
x_{5}=\frac{1}{[(9.0476+0.4926 i)-(9)]} \\
\Rightarrow x_{5}=0.1943-2.0113 i
\end{gathered}
$$

Then $a=0.1943-(0)=0.1943$

$b=-2.0113-(-3)=0.9887$

Therefore $\left[x_{5}\right]=0-3 i+i=-2 i \Rightarrow a_{5}=-2 i$.

$$
\begin{gathered}
x_{6}=\frac{1}{[(0.1943-2.0113 i)-(-2 i)]} \\
\Rightarrow x_{6}=5.1293+0.2983 i
\end{gathered}
$$

Then $a=5.1293-(5)=0.1293$

$b=0.2983-(0)=0.2983$

Therefore $\left[x_{6}\right]=5+0+0=5 \Rightarrow a_{6}=5$.

$$
x_{7}=\frac{1}{[(5.1293+0.2983)-(5)]} \Rightarrow x_{7}=1.2233-2.8221 i
$$

Then $a=1.2233-(1)=0.2233$

and $b=-2.8221-(-3)=0.4012$

Therefore $\left[x_{7}\right]=1-3 i+0=1-3 i \Rightarrow a_{7}=1-3 i$.

$$
\begin{aligned}
& x_{8}=\frac{1}{[(1.2233-2.8221 i)-(1-3 i)]} \\
& \Rightarrow x_{8}=2.7395-2.1825 i
\end{aligned}
$$

Then $a=2.7395-(2)=0.7395$

$b=-2.1825-(-3)=0.8125$

Therefore $\left[x_{8}\right]=2-3 i+i=2-2 i \Rightarrow a_{8}=2-2 i$.
Therefore the continued fraction of the complex number and its conjugate is

$1.1+0.9950 i=\langle 1+i, 10,-1-2 i, 1,9,-2 i, 5,1-3 i, 2-2 i, \cdots\rangle$

and

$1.1-0.9950 i=\langle 1-i, 10-i,-1-2 i, 1,9,-2 i, 5,1-3 i, 2-2 i, \cdots\rangle$

Using theorem we can identify the complex number of the above continued fraction

and

Consider the continued fraction

$[1+i, \quad 10,-1-2 i, \quad 1, \quad 9,-2 i, \quad 5,1-3 i, 2-2 i \cdots]$

Here

$\alpha_{0}=1, \quad \alpha_{1}=10, \quad \alpha_{2}=-1, \quad \alpha_{3}=1, \quad \alpha_{4}=9, \quad \alpha_{5}=0, \ldots$

$\beta_{0}=1, \quad \beta_{1}=0, \quad \beta_{2}=-2, \quad \beta_{3}=0, \quad \beta_{4}=0, \beta_{5}=-2, \ldots$

Then $\quad \frac{p_{0}}{q_{0}}=1+i$

and

$$
q_{0}
$$

$\frac{p_{1}}{q_{1}}=\frac{11+i}{10}=1.1+0.1 i$

$\frac{p_{2}}{q_{2}}=\frac{-9-23 i}{-9-20 i}=1.1247+0.0561 i$

$\frac{p_{3}}{q_{3}}=\frac{2-22 i}{1-20 i}=1.1022+0.0448 i$

$\frac{p_{4}}{q_{4}}=\frac{9-221 i}{-200 i}=1.105+0.045 i$

$\frac{p_{5}}{q_{5}}=\frac{-440-40 i}{-399}=1.1+0.1003 i$

which is approximately equal to the complex number $1.1+0.9950 i$

Next to consider the continued fraction

$[1-i, 10-i,-1-2 i, 1,9,-2 i, 5,1-3 i, 2-2 i \cdots]$

Here

$\alpha_{0}=1, \alpha_{1}=10, \alpha_{2}=-1, \alpha_{3}=1, \alpha_{4}=9, \alpha_{5}=0, \ldots$

$\beta_{0}=-1, \beta_{1}=-1, \beta_{2}=-2, \beta_{3}=0, \beta_{4}=0, \beta_{5}=-2$,

and 


$$
\begin{aligned}
& \text { Then } \quad \frac{p_{0}}{q_{0}}=1-i \\
& \frac{p_{1}}{q_{1}}=\frac{10-11 i}{10-i}=1.0990-0.9900 i \\
& \frac{p_{2}}{q_{2}}=\frac{-31-10 i}{-11-19 i}=1.1016-0.9938 i \\
& \frac{p_{3}}{q_{3}}=\frac{-21-21 i}{-1-20 i}=1.0998-0.9950 i \\
& \frac{p_{4}}{q_{4}}=\frac{-220-199 i}{-20-199 i}=1.0999-0.9950 i \\
& \frac{p_{5}}{q_{5}}=\frac{-419+419 i}{-399+20 i}=1.0999-0.9950 i
\end{aligned}
$$

which is approximately equal to the complex conjugate $1.1-0.9950 i$

\section{CONCLUSION}

Continued fraction method of solving a quadratic equation is a lengthy procedure. In this method the roots may have infinite or periodic representation. Also occurrences of complex roots for a quadratic equation is common. It is well known that complex roots occur in pairs. In this paper, an attempt has been made to represent a complex number and its conjugate in terms of continued fractions. In future, we transform that into finding complex roots of a quadratic equation.

\section{REFERENCES}

1. Arumugam S., Thangapandi Issac A., Somasundaram A., "Complex Analysis", $9^{\text {th }}$ Reprint, Scitech Publications (India) Pvt. Ltd. , December 2007.

2. 2.Ponnusamy S., " Foundations of Complex Analysis", Second edition, Narosa Publishing House, New Delhi.

3. 3.Bosma W., Bastiaan cijsouw, "Complex Continued Fraction Algorithms", Thesis, Radboud University, November 2015.

4. 4.Shalit J.O., Integer Functions and Continued fractions", A.B. Thesis, Princeton University, 1979.

\section{AUTHORS PROFILE}

A.Gnanam, Assistant professor ,Department of Mathematics, Government Arts College, Trichy-22. Email: gnaanam@yahoo.com

S.Krithika, Assistant professor ,Department of Mathematics, Seethalakshmi Ramaswami College, Trichy-2

Email:krithikasrc@gmail.com 